\title{
Electroacupuncture pretreatment induces tolerance against cerebral ischemia/reperfusion injury through inhibition of the autophagy pathway
}

\author{
ZHOUQUAN WU ${ }^{1,2}$, ZHIQING ZOU ${ }^{2}$, RONG ZOU ${ }^{1}$, XIANJU ZHOU $^{3}$ and SUYANG CUI ${ }^{1}$ \\ ${ }^{1}$ The First Clinical College, Nanjing University of Chinese Medicine, Nanjing, Jiangsu 210029; \\ ${ }^{2}$ Laboratory of Neurological Diseases, Department of Anesthesiology; ${ }^{3}$ Department of Neurology, \\ Changzhou No. 2 People's Hospital, The Affiliated Hospital of Nanjing Medical University, \\ Changzhou, Jiangsu 213003, P.R. China
}

Received February 5, 2014; Accepted November 7, 2014

DOI: $10.3892 / \mathrm{mmr} .2015 .3253$

\begin{abstract}
Electroacupuncture (EA) pretreatment has been reported to induce tolerance against cerebral ischemia/reperfusion (I/R) injury; however, the mechanisms underlying the beneficial effects of EA remain to be elucidated. Increasing evidence has suggested that excess activation of autophagy is important in I/R injury. The present study aimed to investigate the hypothesis that EA pretreatment-induced tolerance to cerebral I/R injury was mediated by inhibition of the autophagy pathway. Rats were treated with EA at the acupoint 'Baihui (GV20) $30 \mathrm{~min} / \mathrm{day}$, for five consecutive days prior to the induction of focal cerebral ischemia for 120 min by middle cerebral artery occlusion. Levels of autophagy, cerebral apoptosis, infarct volumes, brain water content and motor deficit were evaluated $12 \mathrm{~h}$ following I/R. The autophagy inducer rapamycin was used to investigate the role of autophagy in mediating neuroprotective effects. The results showed that the number of autophagosomes and the expression of the marker proteins of autophagy, including microtubule-associated protein 1A light chain 3 (LC3)-II and Beclin 1 were significantly increased $12 \mathrm{~h}$ post-I/R. EA pretreatment decreased the expression of autophagy markers and the number of autophagosomes in the ischemic cortex. In addition, EA pretreatment inhibited neuronal apoptosis, reduced infarct volume and
\end{abstract}

Correspondence to: Dr Suyang Cui, The First Clinical College, Nanjing University of Chinese Medicine, 138 Xianlin Avenue, Nanjing, Jiangsu 210029, P.R. China

E-mail: cuisuyang@163.com

Dr Xianju Zhou, Department of Neurology, Changzhou No. 2 People's Hospital, The Affiliated Hospital of Nanjing Medical University, 29 Xinglong Avenue, Changzhou, Jiangsu 213003, P.R. China

E-mail: hippocampus2007@gmail.com

Key words: electroacupuncture, ischemia/reperfusion, autophagy, rapamycin, middle cerebral artery occlusion water content, as well as improved neurological outcome of rats following I/R. Furthermore, the reduced expression of LC3-II and Beclin 1 and the neuroprotective effects were reversed by the autophagy inducer rapamycin. In conclusion, the results of the present study demonstrated that EA pretreatment protected the brain against I/R injury via inhibition of the autophagy process.

\section{Introduction}

As one of the most prevalent causes of human mortality, stroke accounts for hundreds of thousands of mortalities annually worldwide (1). Rapid revascularization of the occluded vessels and timely reperfusion is one of the most effective approaches to salvage cerebral ischemic damage; however, the restoration of blood flow during reperfusion may evoke ischemia/reperfusion (I/R) injury that is not present during ischemia but can be modulated only at reperfusion (2). Efforts have been made to attenuate the injury occurring during reperfusion, including numerous types of pretreatment measurements $(2,3)$. Electroacupuncture (EA) is a novel therapy based on traditional acupuncture in combination with modern electrotherapy (4). Evidence has shown that following vessel occlusion, EA not only reduces myocardial injury but also significantly promotes the recovery of neurological function and thus improves quality of life for patients $(5,6)$. In recent years, several studies have shown that EA pretreatment has beneficial effects, including reperfusion tolerance (7); however, it remains to be elucidated whether the underlying mechanisms of EA require the involvement of autophagy.

Autophagy, a cellular process mediating the lysosomal degradation of long-lived cytoplasmic proteins, is initiated under different conditions, including differentiation, starvation, stress (such as, oxidative or endoplasmic reticulum stress) and protein aggregate accumulation (8-10). During autophagy, cytoplasmic components are sequestered into double-membrane vesicles termed autophagosomes, which then fuse with lysosomes to produce single-membraned autophagosomes and are eventually degraded by lysosomal hydrolases $(11,12)$. There are at least two marked proteins in the autophagic cascade: 
Microtubule-associated protein 1A light chain 3 (LC3), which exists in cytosolic form (LC3-I) and membrane-bound form (LC3-II), the ratio of conversation from LC3-I to LC3-II is closely correlated with the extent of autophagosome formation (13); and Beclin 1, which is essential for recruitment of other autophagic proteins during the expansion of pre-autophagosomal membrane $(14,15)$. Previous studies have reported the autophagy was activated following ischemic injury; however, the contribution of autophagy to neuronal survival/death remains in debate. Autophagy activation maintains cellular homeostasis and survival either through purging the cell of dysfunctional organelles, toxic metabolites and intracellular pathogens, or generating the intracellular building blocks required to preserve vital functions during nutrient deprivation $(16,17)$; for example, the autophagy induced by focal ischemia preconditioning was reported to be beneficial $(18,19)$. Conversely, autophagy also triggers non-apoptotic programmed cell death (autophagic cell death) through excessive self-digestion and degradation of essential cellular constituents, which was implicated in various physiological and pathological conditions associated with neurological diseases (20); for example, autophagy induced by permanent cerebral ischemia was reported to be deleterious (21-23). Overall, physiological levels of autophagy due to mild hypoxia, appear to be protective. By contrast, increased levels of autophagy due to severe hypoxia and/or reperfusion may lead to self-digestion and eventual cell death (24). The present study aimed to investigate the hypothesis that EA exerted neuroprotective effects via inhibition of autophagy during reperfusion, using middle cerebral artery occlusion (MCAO) model rats.

\section{Materials and methods}

The experimental protocol used in the present study was approved by the Ethics Committee for Animal Experimentation of Nanjing University of Chinese Medicine (Nanjing, China), and was conducted according to the Guidelines for Animal Experimentation of Nanjing University of Chinese Medicine. Male Sprague-Dawley rats, weighing 280-320 g, were provided by the Experimental Animal Center of the Nanjing University of Chinese Medicine and were housed under controlled conditions as follows: 12 -h light/dark cycle, $21 \pm 2^{\circ} \mathrm{C}$ and $60-70 \%$ humidity, for a minimum of one week prior to drug treatment or surgery. Rats were provided with a standard rodent diet and tap water ad libitum. The focal ischemia/reperfusion models were established through MCAO (25). During the procedure, room temperature was maintained at $25^{\circ} \mathrm{C}$. All rats were anesthetized with an intraperitoneal injection of sodium pentobarbital (40 mg/kg; Sigma-Aldrich, St. Louis, MO, USA), and an incision was made on the midline of the neck. The right common carotid artery (CCA), the external carotid artery (ECA) and internal carotid artery (ICA) were isolated, and temporarily ligated at their origin with a 6.0 silk suture. Microaneurysm clips were placed around the CCA and CEA to prevent bleeding during insertion of the suture. A hole was subsequently made in the CCA, between the clips with a needle (2-ml injector) and the blunted tip of a nylon suture (diameter, $0.205 \mathrm{~mm}$; length, $20 \pm 2 \mathrm{~mm}$ ) was inserted through the hole until a mild resistance was felt. The two remaining loose collar sutures were gently tightened, and the vessel clips were withdrawn. Rats were anesthetized using an intraperitoneal injection of $40 \mathrm{mg} /$ $\mathrm{kg}$ sodium pentobarbital while undergoing EA stimulation or surgery.

Experiment protocol. In order to determine the time-dependent effects of $\mathrm{I} / \mathrm{R}$ on autophagy expression, rats were assigned at random to one of the following four groups: Sham, $6 \mathrm{~h}$ following I/R (I/R 6 h), I/R 12 h, and I/R 24 h. The optimum time-point was determined and rats were then assigned to one of the following five groups: Sham, I/R $12 \mathrm{~h}, \mathrm{EA}+\mathrm{I} / \mathrm{R} 12 \mathrm{~h}$, Rapamycin+ EA+I/R 12 h (Sigma-Aldrich) or Vehicle+EA+I/R 12 h (Fig. 1). The rats in the sham group underwent an identical surgical procedure without MCAO. I/R was established through MCAO for $120 \mathrm{~min}$ followed by reperfusion for $12 \mathrm{~h}$; EA pretreatment was achieved through stimulation of the acupoint 'Baihui (GV20)' on five consecutive days prior to I/R. Levels of autophagy were determined using transmission electron microscopy and western blot analysis. The neuroprotective effects of EA were determined using a terminal deoxynucleotidyl transferase-mediated deoxyuridine-triphosphatase nick end labeling (TUNEL) assay, infarct volumes, brain water content and motor deficit. The numbers of rats used for each experiment are summarized in Table I.

EA pretreatment. EA pretreatment was performed at the acupoint 'Baihui (GV20)' (5). The acupoint 'Baihui' is located at the intersection of the sagittal midline and the line between the two ears (26). Animals were anesthetized and stimulated at an intensity of $1 \mathrm{~mA}$ and a frequency of $2 / 15 \mathrm{~Hz}$ for $30 \mathrm{~min} /$ day for five consecutive days prior to MCAO, using the Hwato Electronic Acupuncture Treatment Instrument (SDZ-V; Suzhou Medical Appliances Co., Ltd., Jiangsu, China).

Transient focal cerebral ischemia. Rats were subjected to transient MCAO for 120 min following the final pretreatment as described above. Reperfusion was achieved by withdrawing the suture following $120 \mathrm{~min}$ ischemia. Cerebral blood flow (CBF) through the middle cerebral artery was measured using laser Doppler flowmetry (PeriFlux 5000; Perimed, Järfälla, Sweden). A flexible fiber-optic probe (PeriFlux 5000; Perimed) was affixed to the skull over the cortex supplied by the proximal part of the middle cerebral artery $(2 \mathrm{~mm}$ caudal to bregma and $6 \mathrm{~mm}$ lateral to middle). Animals with $<80 \%$ reduction in $\mathrm{CBF}$ in the core of the middle cerebral artery area were excluded from this study.

Treatment with the autophagy inducer. In order to determine whether rapamycin, an autophagy inducer, attenuated the neuroprotective effects of EA at the acupoint 'Baihui (GV20)', rats were treated with intracerebral ventricle injection (i.c.v.) of $35 \mathrm{pmol}$ rapamycin (Sigma, St. Louis, MO, USA) at the onset of reperfusion; the induction of autophagy using 35 pmol rapamycin was significant according to previous studies (19). Rapamycin was dissolved in ethanol and then diluted to the final concentration using normal saline solution (final ethanol concentration, $<2 \%$ ). Control animals received the same volume injection of vehicle.

Transmission electron microscopic examination. Following reperfusion for $12 \mathrm{~h}$, rats were anesthetized, as described above, and then perfused with pre-cooled phosphate-buffered saline (PBS; pH 7.4) followed by PBS containing 4\% paraformaldehyde 
Table I. Experimental protocol.

\begin{tabular}{|c|c|c|c|c|c|}
\hline Protocol & Experiment & Time-point & Group & Number & Total \\
\hline \multirow[t]{2}{*}{$\begin{array}{l}\text { Effect of EA on } \\
\text { autophagy expression }\end{array}$} & $\begin{array}{l}\text { Transmission electron } \\
\text { microscopic }\end{array}$ & $\mathrm{I} / \mathrm{R} 12 \mathrm{~h}$ & $\begin{array}{l}\text { sham, I/R } 12 \mathrm{~h}, \\
\text { EA +I/R } 12 \mathrm{~h}, \\
\text { Rapamycin+EA+ } \\
\text { I/R } 12 \mathrm{~h}, \text { Vehicle } \\
+\mathrm{EA}+\mathrm{I} / \mathrm{R} 12 \mathrm{~h}\end{array}$ & $\mathrm{n}=5$ & 25 \\
\hline & Western blotting & $\mathrm{I} / \mathrm{R} 12 \mathrm{~h}$ & $\begin{array}{l}\text { sham, I/R } 12 \mathrm{~h}, \\
\text { EA +I/R } 12 \mathrm{~h}, \\
\text { Rapamycin+EA } \\
+\mathrm{I} / \mathrm{R} 12 \mathrm{~h}, \text { Vehicle+EA } \\
\text { +I/R } 12 \mathrm{~h}\end{array}$ & $\mathrm{n}=5$ & 25 \\
\hline \multirow[t]{2}{*}{$\begin{array}{l}\text { Neuroprotective } \\
\text { effect of EA }\end{array}$} & TUNEL assay & $\mathrm{I} / \mathrm{R} 12 \mathrm{~h}$ & $\begin{array}{l}\text { sham, I/R } 12 \mathrm{~h}, \\
\text { EA +I/R } 12 \mathrm{~h}, \\
\text { Rapamycin+EA+ } \\
\text { I/R } 12 \mathrm{~h}, \text { Vehicle +EA } \\
+\mathrm{I} / \mathrm{R} 12 \mathrm{~h}\end{array}$ & $\mathrm{n}=5$ & 25 \\
\hline & TTC staining & $\mathrm{I} / \mathrm{R} 12 \mathrm{~h}$ & $\begin{array}{l}\mathrm{I} / \mathrm{R} 12 \mathrm{~h}, \\
\mathrm{EA}+\mathrm{I} / \mathrm{R} 12 \mathrm{~h}, \\
\text { Rapamycin+EA+I/R } 12 \mathrm{~h}\end{array}$ & $\mathrm{n}=8$ & 24 \\
\hline
\end{tabular}

Sum

EA, electroacupuncture; I/R $12 \mathrm{~h}$, ischemia/reperfusion for $12 \mathrm{~h}$; TUNEL, terminal deoxynucleotidyl transferase-mediated deoxyuridine-triphosphatase nick end labeling; TTC, 2,3,5-triphenyltetrazolium chloride.

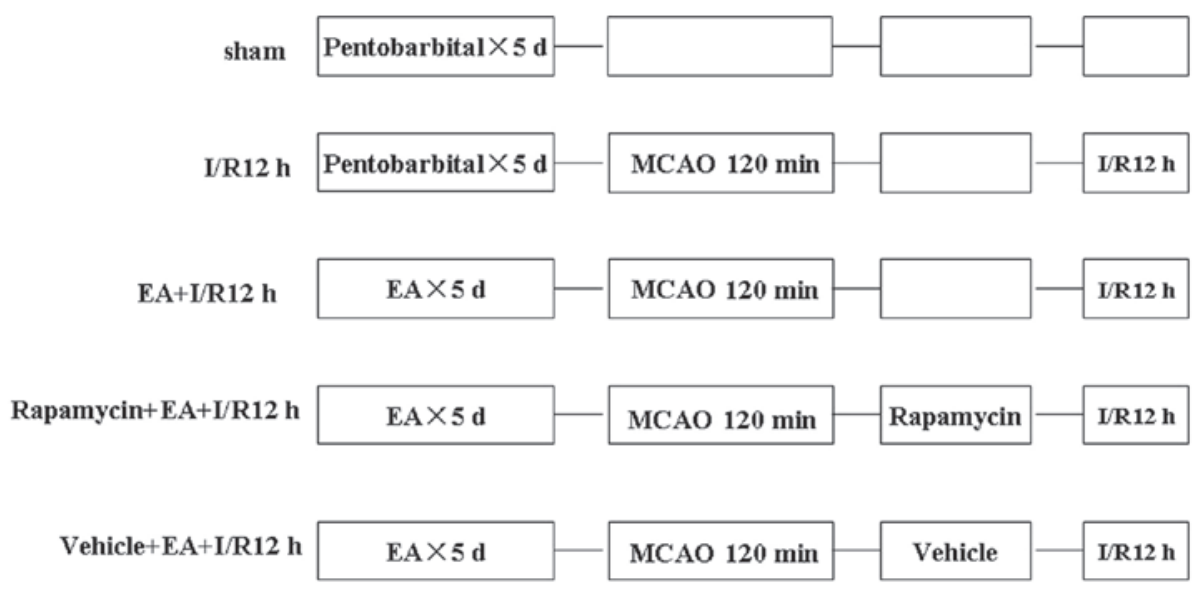

Figure 1. Experimental procedure used to evaluate the role of autophagy in EA-induced neuroprotection. Treatment groups were as follows: Sham, rats underwent an identical surgical procedure without MCAO; I/R $12 \mathrm{~h}$ : I/R was established by MCAO for 120 min followed by reperfusion for $12 \mathrm{~h} ; \mathrm{EA}+\mathrm{I} / \mathrm{R} 12 \mathrm{~h}, \mathrm{EA}$ pretreatment through stimulating the acupoint 'Baihui (GV20)' for five consecutive days prior to I/R; Rapamycin+EA+I/R $12 \mathrm{~h}$ and Vehicle+EA+I/R $12 \mathrm{~h}$, rats injected intracerebrally with $35 \mathrm{pmol}$ rapamycin or vehicle at the onset of reperfusion. EA, electroacupuncture pretreatment; MCAO, middle cerebral artery occlusion; I/R $12 \mathrm{~h}$, ischemia/reperfusion for $12 \mathrm{~h}$.

and $0.25 \%$ glutaraldehyde. The brains were removed and kept overnight in a solution of $2 \%$ paraformaldehyde and $2.5 \%$ glutaraldehyde in 0.1 M PBS ( $\mathrm{pH}$ 7.4). The following day, the rat brains were sectioned using a vibratome (myNeuroLab; Leica Microsystems, Ltd, Milton Keynes, UK) into 0.5-mm slices. The parietal lobe cortex in the ischemic core area was selected for analysis, and selected areas were processed by post-fixation in $1 \%$ osmium tetroxide for $1 \mathrm{~h}$, dehydrated in graded ethanol and then embedded in epoxy resin. Polymerization was performed at $80^{\circ} \mathrm{C}$ for $24 \mathrm{~h}$. Blocks were sectioned using an PowerTome-PC ultramicrotome (RMC, Inc., Tucson, AZ, USA) into ultrathin sections $(60-70 \mathrm{~nm})$, which were post-stained with uranyl acetate and lead citrate, and visualized using a Hitachi 7100 electron microscopy (Nikon, Tokyo, Japan). For quantitative analysis of the number of autophagosomes, ten fields of vision for each rat (three rats in each group) were examined as previously described (27).

TUNEL assay. A TUNEL assay (Boster Biological Technology, Ltd, Wuhan, China) was performed according to the 
manufacturer's instructions. Brain sections were stained with diaminobenzidine, counterstained with hematoxylin, dehydrated with gradient alcohol, cleared with xylene and then mounted with neutral resin. The reaction mixture was replaced with PBS in the negative control. Normal nuclei were stained blue and apoptotic nuclei were stained brownish-black or brown. Four sections were observed from each rat (magnification, $\mathrm{x} 400$ ) and ten fields of vision $(n=5)$ on each slide were counted. The percentage of positive apoptotic nuclei in the total number of cells per field was calculated and the mean value was taken to be the apoptotic index of cerebral neurons.

Western blotting. Following reperfusion for $12 \mathrm{~h}$, the ischemic parietal lobe cortex supplied by the right MCAO and the corresponding area of sham-operated rats were homogenized and proteins were extracted using a lysis buffer $(10 \mathrm{mM}$ Tris-Hcl, pH 7.4; 150 mM NaCl; $1 \%$ Triton X-100; 0.1\% SDS; 5 mM EDTA; $1 \mathrm{mM}$ PMSF; $0.28 \mathrm{U} / \mathrm{ml}$ aprotinin; $50 \mu \mathrm{g} / \mathrm{ml}$ leupeptin; $1 \mathrm{mM}$ benzamidine; and $7 \mu \mathrm{g} / \mathrm{ml}$ pepstatin A) as previously described (28). Protein concentrations were determined using a spectrophotometer (UV-2540; SHIMADZH Corp., Kyoto, Japan). A $60 \mu \mathrm{g}$ aliquot of protein from each sample was separated using $10 \%$ SDS-PAGE and subsequently transferred to a nitrocellulose membrane (Shanghai Haoran Bio-Technology Co., Ltd, Shanghai, China). The membranes were then incubated with specific polyclonal antibodies against LC3 (\#4108; 1:1,000; Cell Signaling Technology, Inc., Danvers, MA, USA), or Beclin 1 (\#3738; 1:500; Cell Signaling Technology, Inc.) at $4^{\circ} \mathrm{C}$ over night, and then incubated with a horseradish peroxidase-conjugated goat anti-rabbit secondary antibody (sc2004; 1:5,000; Jackson ImmunoResearch Laboratories, Inc., West Grove, PA, USA) at room temperature for $1 \mathrm{~h}$. Immunoreactivity was detected using enhanced chemiluminescent autoradiography (Bioroc Pharmaceutical \& Biotech Co., Ltd, Tianjin, China) in accordance with the manufacturer's instructions. The membranes were reprobed with $\beta$-actin (1:5,000; Santa Cruz Biotechnology, Inc., Dallas, TX, USA) following stripping. Films were then used for final determination of protein expression using Sigma scan software (scanPDF15; Sigma-Aldrich) and normalized to the loading control.

Evaluation of infarct volume, brain water content and motor deficits. Following reperfusion for $12 \mathrm{~h}$, the motor deficits in rats were evaluated in a blinded manner using a previously described protocol (29) and were scored as follows: 0, Rats behave normally; 1 , rats cannot fully stretch their left front leg; 2 , rats turn around in a circle; 3 , rats fall down to the left side; 4 , rats cannot move by themselves and lose consciousness. Following scoring, rats with $>2$ points were sacrificed by anesthetization with sodium pentobarbital followed by decapitation, and the brains were dissected and sliced in a plastic module (3-mm) and then stained with 2\% 2,3,5-triphenyltetrazolium chloride (TTC) for $30 \mathrm{~min}$. The total wet weight of the TTC-stained brains was quantified using an electronic scale. The wet red and white brain regions of the TTC-stained brains were collected separately. Infarct volume was analyzed using five slices of 3-mm coronal sections from each brain and calculated with the following formula: Infarct volume $(\%)=[$ (total wet weight - red weight)/total wet weight] $\mathrm{x} 100$. The red and white sections of these brains were then desiccated at $105^{\circ} \mathrm{C}$ for $48 \mathrm{~h}$ until the weight was constant. The total weight of the dried TTC-stained brains was follows: Water content $(\%)=[($ wet weight-dried weight $) /$ wet weight $] \times 100(30)$.

Statistical analysis. Statistical analysis was performed using a one-way analysis of variance followed by a Student-Newman-Keul's post hoc analysis. Statistical analyses were performed using SPSS 15.0 (SPSS, Inc., Chicago, IL, USA). $\mathrm{P}<0.05$ was considered to indicate a statistically significant difference between values.

\section{Results}

Autophagy is upregulated following I/R. In order to determine the time-dependent effects of I/R on autophagy, the expression levels of the activated autophagy biomarker LC3 were detected using western blot analysis and the number of autophagosomes present were observed using transmission electron microscopic at different time points following I/R. In addition, the ratio of LC3-II/LC3-I was determined, which reflected the extent of autophagosome formation. As shown in Fig. 2A, the ratio of LC3-II/LC3-I in the ischemic cortex of adult rats for each group were $0.19,0.5,0.7$ and 0.34 , respectively. The ratio at each time-point (6-24 h) following I/R was significantly increased compared to that of the sham group $(\mathrm{F}=18.63, \mathrm{P}=0.000)$, with a maximal value at $12 \mathrm{~h}$ following I/R. Similarly, the number of autophagosomes significantly increased at each time-point following $\mathrm{I} / \mathrm{R}(\mathrm{F}=48.44, \mathrm{P}=0.000)$, and peaked at $12 \mathrm{~h}$ (Fig. 2B). Therefore $12 \mathrm{~h}$ post-I/R was used for all subsequent experiments to further explore the potential autophagic mechanism of EA pretreatment.

EA pretreatment decreases the autophagosome and cerebral apoptosis $12 \mathrm{~h}$ after I/R. In order to examine the direct effect of EA pretreatment on autophagy, transmission electron microscopy was used to observe the morphological changes of neurons in each treatment group. Neurons in the cortex of the sham group appeared to be normal with comparatively healthy endoplasmic reticulum, mitochondria, lysosomes and nuclei (Fig. 3A). By contrast, neurons in the I/R 12 h group displayed an apparent disease morphology; the neurons showed fragmented endoplasmic reticulum, formation of numerous vacuoles in the cytoplasm and condensation of chromatin. Numerous autophagosomes (C-shaped double-membrane structures) and lysosomes with engulfed cytoplasmic materials were observed. Neurons in the $E A+I / R ~ 12 ~ h$ group displayed mild injury, with a number of normal organelles and nuclei observed; however, autophagosomes remained present. Quantitative analysis of the electron microscopy images showed that the number of autophagosomes in EA+I/R 12 h group was significantly decreased compared with that of the I/R $12 \mathrm{~h}$ group $(0.098$ and 0.05 autophagosomes per $\mu \mathrm{m}^{2}$, respectively; $\left.\mathrm{P}<0.05\right)$.

In order to determine the contribution of autophagic mechanisms to the beneficial effect elicited through EA pretreatment, a single i.c.v injection of the autophagy inducer rapamycin $(35 \mathrm{pmol})$ was administered at the onset of reperfusion and its effects were examined. The results showed that neurons in the rapamycin + EA $+\mathrm{I} / \mathrm{R} 12 \mathrm{~h}$ group exhibited injury comparable to that of the I/R $12 \mathrm{~h}$ group, 
$\mathbf{A}$
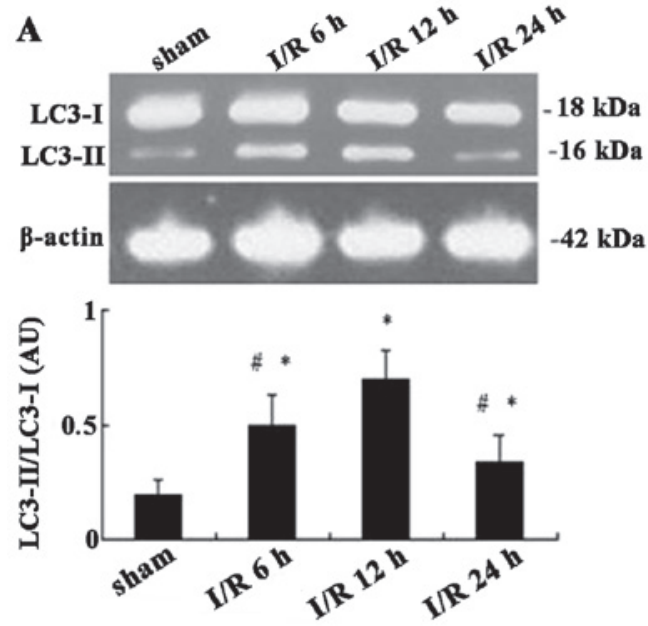

B

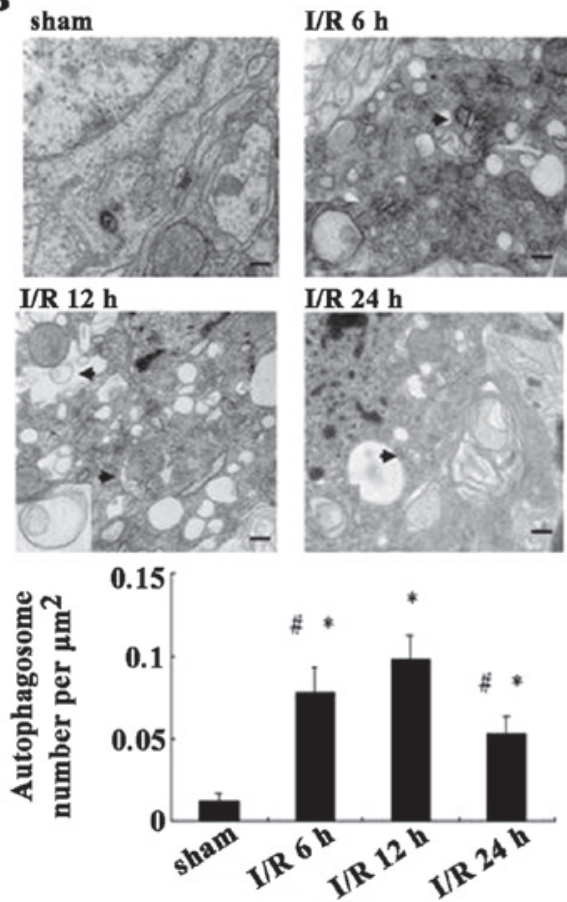

Figure 2. Autophagy is upregulated following I/R. Rats were subjected to $120 \mathrm{~min}$ ischemia and sacrificed at different reperfusion times (6, 12 and $24 \mathrm{~h})$ (A) Upper panel: Western blot analysis of the autophagy marker protein LC3 at each time-point. Lower panel: Quantitative analysis of the ratio LC3-II/LC3-I. $\beta$-actin was used as a loading control. (B) Upper panel: Representative electron microscopy images of rat brains stained with uranyl acetate and lead citrate for each time-point, arrows indicate autophagosomes. Lower panel: Quantitative analysis of the number of autophagosomes observed per $\mu \mathrm{m}^{2}$. Ten fields of vision were observed for each rat $(\mathrm{n}=5)$. Scale bar, $0.5 \mu \mathrm{m})$. Values are presented as the mean \pm standard deviation. " $\mathrm{P}<0.05$ vs. sham group, ${ }^{\#} \mathrm{P}<0.05 \mathrm{vs}$. I/R $12 \mathrm{~h}$ group. $\mathrm{I} / \mathrm{R}$, ischemia/reperfusion; LC3, microtubule-associated protein 1A light chain 3.

suggesting that rapamycin suppressed the beneficial effect of EA pretreatment.

Furthermore, cerebral apoptosis was detected using a TUNEL assay. As shown in Fig. 3B, cerebral apoptosis was significantly increased in rats subjected to I/R $12 \mathrm{~h}$ compared to that of the sham group, whereas EA pretreatment significantly inhibited I/R-induced apoptosis (32.1 and 15.5\%, respectively; $\mathrm{P}<0.05)$. However, following administration with 35 pmol rapamycin prior to the onset of reperfusion, levels of cerebral apoptosis were comparable to that of the I/R 12 h group, therefore attenuating the effects of EA pretreatment.

These results therefore supported the hypothesis that EA pretreatment exerted a protective effect during I/R through suppression of neuronal autophagy.

EA pretreatment reduces the expression of $I / R$-induced autophagic marker proteins $12 \mathrm{~h}$ following IR. In order to further investigate whether EA pretreatment affects autophagy, western blot analysis was used to detect the expression levels of autophagic marker proteins, LC3-I, LC3-II and Beclin-1 in the ischemic cortex of rats. As shown in Fig. 4A, the expression levels of LC3-II were low in sham group and high in the I/R $12 \mathrm{~h}$ group. EA pretreatment markedly decreased the expression of LC3-II; however, rapamycin attenuated the inhibitory effect of EA on LC3-II. In addition, LC3-I expression only fluctuated marginally among the treatment groups, therefore the ratio of LC3-II/LC3-I was quantified. EA pretreatment significantly decreased the ratio of LC3-II/LC3-I compared with that of the
I/R 12 h group ( 0.55 and 0.87 , respectively; $\mathrm{P}<0.05$ ); however, rapamycin inhibited the decreased ratio of LC3-II/LC3-I in the $\mathrm{EA}+\mathrm{I} / \mathrm{R} 12 \mathrm{~h}$ group ( 0.85 and 0.55 , respectively), with comparable results to that of the I/R $12 \mathrm{~h}$ group. As shown in Fig. 4B, the effect of the treatment groups on Beclin-1 expression was comparable to that on LC3-II expression. Beclin-1 expression was significantly upregulated following I/R and suppressed by EA pretreatment; however, rapamycin attenuated the effects of EA pretreatment on Beclin-1 expression $(\mathrm{F}=8.86, \mathrm{P}=0.000$ vs. $\mathrm{EA}+\mathrm{I} / \mathrm{R} 12 \mathrm{~h}$ group). These results therefore suggested that EA pretreatment prevented I/R-induced autophagy via downregulation of LC3-II and Beclin-1 expression.

Autophagy inducer rapamycin abolishes the neuroprotective effects of EA pretreatment. In order to explore the contribution of autophagic mechanisms to the neuroprotection elicited by EA pretreatment, the effects of rapamycin on infarct volume, water content and motor deficits induced by EA pretreatment were examined (Fig. 5). In rats subjected to $\mathrm{I} / \mathrm{R}$, extensive infarction was detected in the ipsilateral cerebral cortical and subcortical areas over a series of brain sections. EA pretreatment significantly reduced the infarct volume and water content of rats in the I/R $12 \mathrm{~h}$ group, whereas administration of 35 pmol rapamycin abolished the protective effects of EA pretreatment, as evidenced by the increased infarct volume and water content $(\mathrm{F}=10.24 / \mathrm{P}=0.001$ and $\mathrm{F}=11.6 / \mathrm{P}=0.000$, respectively) (Fig. 5A and $\mathrm{B}$ ). Furthermore, the effects of EA pretreatment on I/R-induced motor behavior deficits were evaluated (Fig. 5C); motor deficits were scored using a 


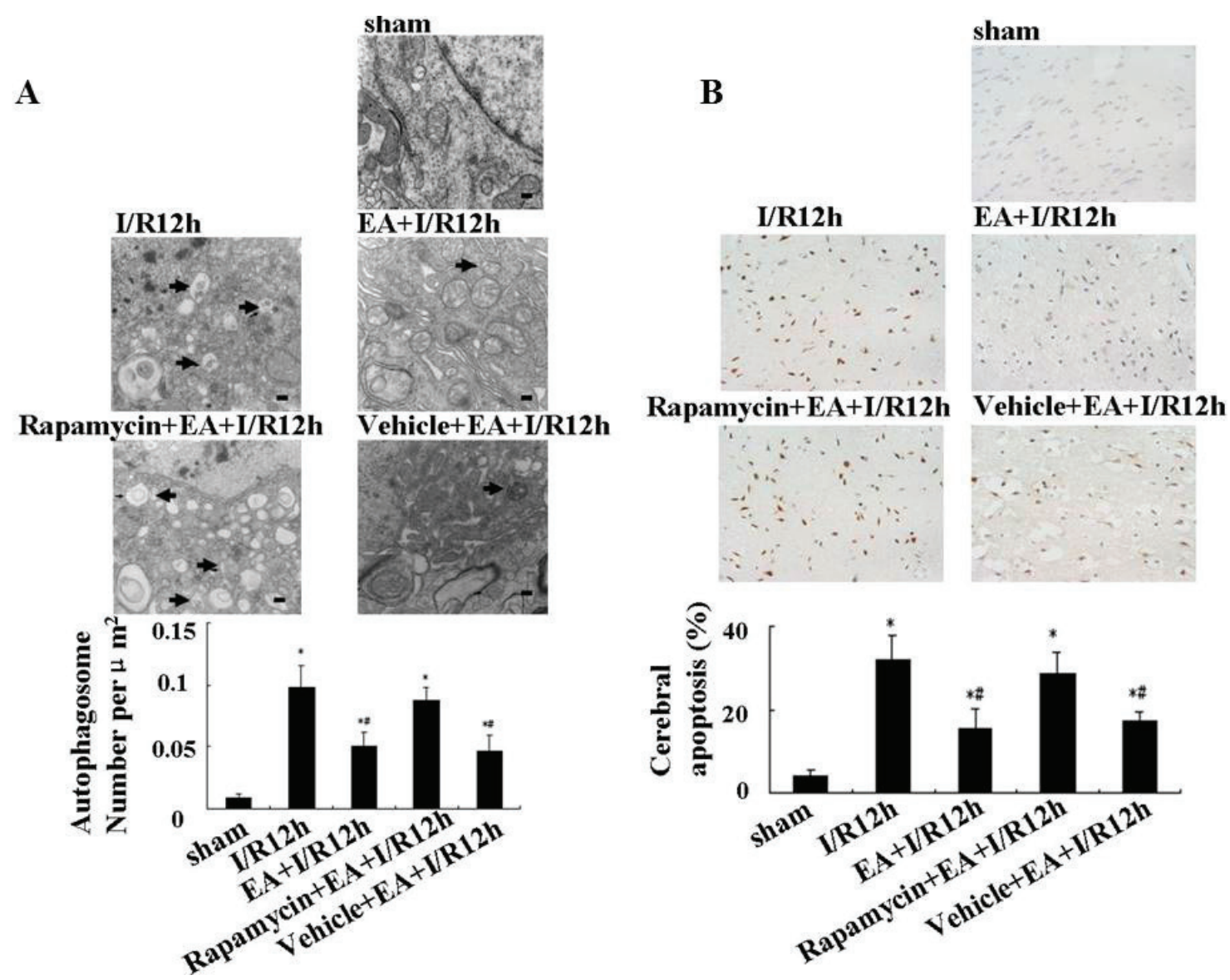

Figure 3. EA pretreatment decreases the number of autophagosomes and cerebral apoptosis $12 \mathrm{~h}$ following I/R. Rats were sacrificed $12 \mathrm{~h}$ following reperfusion. (A) Upper panel: Representative electron microscopy images for each group, arrows indicate autophagosomes. Lower panel: Quantitative analysis of the number of autophagosomes observed per $\mu \mathrm{m}^{2}$. Ten fields of vision were examined for each rat (n=5/group). (B) A terminal deoxynucleotidyl transferase-mediated deoxyuridine-triphosphatase nick end labeling assay was performed in order to determine levels of cerebral apoptosis. Upper panel: Representative images of the stained rate brains: Brown nuclei represent apoptotic cells (magnification, $\mathrm{x} 400$ ). Lower panel: Quantitative analysis for the percentage of cerebral apoptosis. Ten fields of vision were observed for each rat $(\mathrm{n}=5)$. Values are presented as the mean \pm standard deviation. ${ }^{*} \mathrm{P}<0.05$ vs. sham group, ${ }^{"} \mathrm{P}<0.05 \mathrm{vs}$. I/R $12 \mathrm{~h}$ group. EA, electroacupuncture; I/R $12 \mathrm{~h}$, ischemia/reperfusion for $12 \mathrm{~h}$.
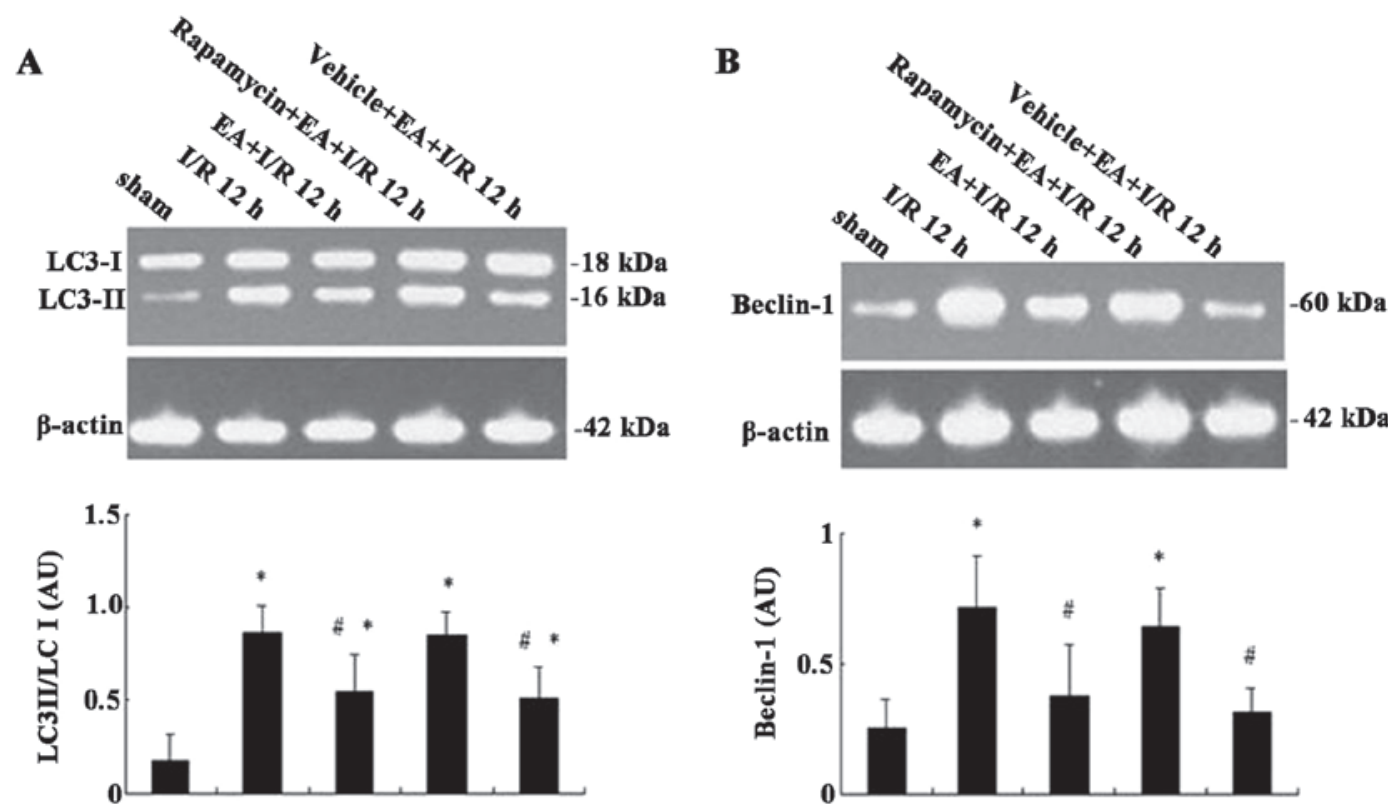

Figure 4. EA pretreatment reduces the expression of LC3-II and Beclin-1. Extracts from the sham and ischemic cerebral cortex were separated for western blot analysis of autophagy marker protein expression. (A) Representive blots for LC3 expression in each group and quantitative analysis of the ratio of LC3-II/LC3-I. (B) Representative blots for Beclin-1 expression in each group and quantitative analysis of Beclin-1 expression. $\beta$-actin was used as the loading control. Values are presented as the mean \pm standard deviation $(\mathrm{n}=5)$. ${ }^{\prime} \mathrm{P}<0.05$ vs. sham group, ${ }^{\text {}} \mathrm{P}<0.05$ vs. IR $12 \mathrm{~h}$ group. EA, electroacupuncture; I/R, ischemia/reperfusion; LC3, microtubule-associated protein 1A light chain 3. 

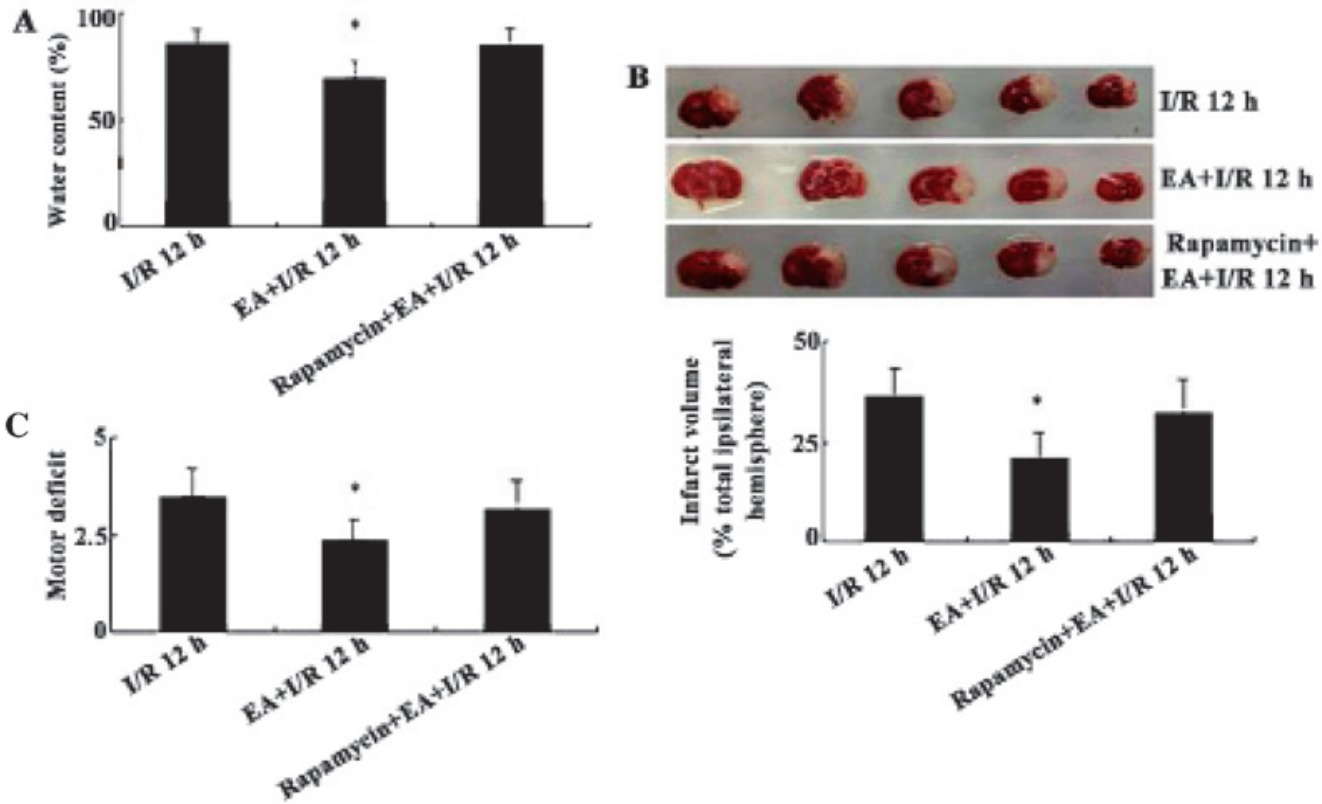

Figure 5. EA pretreatment reduces water content and brain infarct, as well as improves motor deficit following I/R. (A) Measurement of brain edema from each group $12 \mathrm{~h}$ following I/R. (B) Upper panel: Representative infarcts with 2,3,5-triphenyltetrazolium chloride staining for each group. Lower panel: Quantification of infarct volume for each group $12 \mathrm{~h}$ post-reperfusion. (C) Neurological deficit scores for each group $12 \mathrm{~h}$ following I/R. Values are presented as the mean \pm standard deviation ( $\mathrm{n}=8$ for each group). ${ }^{\mathrm{P}} \mathrm{P}<0.05$ vs. I/R $12 \mathrm{~h}$ group. EA, electroacupuncture; I/R, ischemia/reperfusion.

five-point scale as described in the Materials and methods. EA pretreatment $(\mathrm{EA}+\mathrm{I} / \mathrm{R} 12 \mathrm{~h})$ resulted in a significant reduction in motor deficits induced by $\mathrm{I} / \mathrm{R}(\mathrm{F}=6.253, \mathrm{P}=0.007 \mathrm{vs}$. $\mathrm{I} / \mathrm{R}$ $12 \mathrm{~h}$ group), while administration with rapamycin significantly attenuated the beneficial effects of EA pretreatment on neurological motor deficits. These results supported the hypothesis that EA pretreatment protected neurons against I/R through an autophagic mechanism.

\section{Discussion}

EA is a novel combination of traditional acupuncture-based therapy and modern electrotherapy. EA is conducted by inserting acupuncture needles into acupoints and then altering electric stimulation parameters, including stimulation frequency, current intensity, pulse width and pulse interval (31). Therefore, the choice of acupoint and stimulation parameters is important; based on meridian theory, the 'Baihui' acupoint (one of the acupoints of Du merdian) was selected for use in the present study as it receives projections from the motor and sensory cortex, as well as from the anterior cerebral artery. In addition, according to the results of a previous study, 'Baihui acupoint (GV20)'-stimulation with a density-sparse wave of $2 / 15 \mathrm{~Hz}$ and an intensity of $1 \mathrm{~mA}$ for $30 \mathrm{~min} /$ day over five consecutive days was reported to induce cerebral I/R tolerance (32). Evidence has suggested that I/R disturbs energy metabolism, increases oxidative stress and triggers apoptotic cell death (33). Previous studies have reported that EA pretreatment exerted neuroprotective effects against I/R injury via regulation of oxidative stress, maintenance of blood-brain barrier integrity and inhibition of apoptosis via various receptors $(34,35)$. In addition, I/R was reported to induce autophagic cell death $(36,37)$; therefore, it is possible that EA pretreatment protected neurons against $\mathrm{I} / \mathrm{R}$ injury through regulating the levels of autophagy. The present study provided evidence to suggest that the neuroprotective effect of EA pretreatment was, at least in part, associated with the autophagy pathway.

Autophagy is an evolutionarily conserved pathway, which involves the isolation and transport of cytoplasmic materials to lysosomes where proteins and organelles are degraded and recycled. Under physiological conditions, autophagy activation has been associated with neuroprotection via degradation of misfolded protein; for example, preconditioning ischemia protects neurons against subsequent brain damage via upregulated autophagy (38). However, under certain pathological conditions, for example reperfusion following prolonged ischemia, autophagy overactivation promotes cell death through apoptotic-dependent and -independent cascades. A previous study indicated that excess autophagy activation was involved in several models of cerebral ischemia, including global cerebral ischemia, focal ischemia and hypoxia-ischemia, through inducing autophagic cell death (39). Rami et al (23) demonstrated that markedly elevated levels of autophagy contributed to subsequent neuronal death in the penumbra neurons of adults rats following focal cerebral I/R. In addition, Liu et al (40) reported that upregulation of LC3-II was associated with brain injury in post-ischemic brain tissue following global cerebral I/R. Furthermore, Xu et al (41) supported the association between significantly increased levels of the autophagic marker proteins, LC3-II and Beclin-1, and neuronal death in the ischemic cortex of mice following focal cerebral I/R. Consistent with these studies, the results of the present study demonstrated that the ratio of LC3-II/LC3-I and Beclin-1 expression in the ischemic cortex of adult rats increased significantly following focal cerebral ischemia, which may be associated with neuronal damage. These results therefore indicated that autophagy levels may be associated with neuronal fate. 
The results of the present study demonstrated that EA pretreatment exerted significant protective roles in a rat model of focal cerebral ischemic model (MCAO), including the reduction of cerebral apoptosis, infarct volumes and brain edema, which in turn improved motor deficits. These results were concurrent a previous report (7). Notably, the protective effects of EA pretreatment were associated with inhibition of the autophagy pathway, as evidenced by a reduced number of autophagosomes in the ischemic parietal lobe and the attenuated expression of the autophagy marker proteins LC3-II and Beclin-1 at $12 \mathrm{~h}$ following I/R. These results demonstrated that the neuroprotective effects of EA pretreatment may be, in part, associated with the inhibition of autophagy. Furthermore, the effects of the autophagy inducer rapamycin (42) on the neuroprotective effects of EA pretreatment were investigated. The results showed that the neuroprotective effects induced by EA pretreatment were partially reversed by rapamycin, which therefore suggested that the inhibition of autophagic pathways may partially underlie the mechanisms by which EA pretreatment induced tolerance to cerebral I/R injury.

The role of autophagy in neuronal death during reperfusion remains to be fully elucidated. Accumulating evidence has suggested that autophagy activation contributed to ischemic neuronal injury following cerebral I/R $(25,43)$; however, one study supported the opposite conclusion, that autophagy activation was associated with protective effects against neuronal injury (44). For example, Zhang et al (44) evaluated the expression of the autophagic marker LC3-II in the penumbral region $0,1,12$, and $24 \mathrm{~h}$ post-reperfusion and found that levels of LC3-II/GAPDH increased with reperfusion time, and persisted until $24 \mathrm{~h}$; however, the inhibition of autophagy during reperfusion enhanced I/R injury (44). The inconsistency may be due to different ischemic models, durations of the ischemic insults and ages of animals. It was reported that autophagy was more pronounced in adult mice than neonatal mice subjected to hypoxia-ischemia injury, indicating that autophagic cell death may be more significant in mature animals (45). Furthermore, the different durations of the ischemic insults may affect the role of autophagy during reperfusion.

In conclusion, the results of the present study suggested that the neuroprotection induced by EA pretreatment against I/R injury was mediated, at least in part, through inhibition of the autophagy pathway. Therefore, EA may provide a promising novel strategy for the clinical treatment of I/R injury.

\section{Acknowledgements}

The present study was supported by Jiangsu Province Hospital of Traditional Chinese Medicine, The Affiliated Hospital of Nanjing University of Chinese Medicine (project nos. Y12016, CJ20140038 and 81202802).

\section{References}

1. Donnan GA, Fisher M, Macleod M and Davis SM: Stroke. Lancet 371: 1612-1623, 2008

2. Zhao H, Sapolsky RM and Steinberg GK: Interrupting reperfusion as a stroke therapy: ischemic postconditioning reduces infarct size after focal ischemia in rats. J Cereb Blood Flow Metab 26: 1114-1121, 2006.
3. Wang JK, Yu LN, Zhang FJ, Yang MJ, Yu J, Yan M and Chen G: Postconditioning with sevoflurane protects against focal cerebral ischemia and reperfusion injury via PI3 K/Akt pathway. Brain Res 1357: 142-151, 2010.

4. Chou P, Chu H and Lin JG: Effects of electroacupuncture treatment on impaired cognition and quality of life in Taiwanese stroke patients. J Altern Complement Med 15: 1067-1073, 2009.

5. Wang Q, Xiong L, Chen S, Liu Y and Zhu X: Rapid tolerance to focal cerebral ischemia in rats is induced by preconditioning with electroacupuncture: window of protection and the role of adenosine. Neurosci Lett 381: 158-162, 2005.

6. Wang Q, Peng Y, Chen S, Gou X, Hu B, Du J, Lu Y and Xiong L: Pretreatment with electroacupuncture induces rapid tolerance to focal cerebral ischemia through regulation of endocannabinoid system. Stroke 40: 2157-2164, 2009.

7. Wang Q, Wang F, Li X, Yang Q, Xu N, Huang Y, Zhang Q, Gou X, Chen S and Xiong L: Electroacupuncture pretreatment attenuates cerebral ischemic injury through alpha7 nicotinic acetylcholine receptor-mediated inhibition of high-mobility group box 1 release in rats. J Neuroinflammation 9: 24, 2012.

8. Mizushima N: Autophagy: process and function. Genes Dev 21: 2861-2873, 2007.

9. Yorimitsu T, Nair U, Yang Z and Klionsky DJ: Endoplasmic reticulum stress triggers autophagy. J Biol Chem 281: 30299-30304, 2006.

10. Sarkar S, Perlstein EO, Imarisio S, Pineau S, Cordenier A, Maglathlin RL, Webster JA, Lewis TA, et al: Small molecules enhance autophagy and reduce toxicity in Huntington's disease models. Nat Chem Biol 3: 331-338, 2007.

11. Shintani T and Klionsky DJ: Autophagy in health and disease: a double-edged sword. Science 306: 990-995, 2004.

12. Mizushima N: The pleiotropic role of autophagy: from protein metabolism to bactericide. Cell Death Differ 12 Suppl 2: 1535-1541, 2005.

13. Kabeya Y, Mizushima N, Ueno T, Yamamoto A, Kirisako T, Noda T, Kominami E, Ohsumi Y and Yoshimori T: LC3, a mammalian homologue of yeast Apg8p, is localized in autophagosome membranes after processing. EMBO J 19: 5720-5728, 2000.

14. Liang XH, Jackson S, Seaman M, Brown K, Kempkes B, Hibshoosh $\mathrm{H}$ and Levine B: Induction of autophagy and inhibition of tumorigenesis by beclin 1. Nature 402: 672-676, 1999.

15. Pattingre S, Tassa A, Qu X, Garuti R, Liang XH, Mizushima N, Packer M, Schneider MD and Levine B: Bcl-2 antiapoptotic proteins inhibit Beclin 1-dependent autophagy. Cell 122: 927-939, 2005.

16. Qin ZH, Wang Y, Kegel KB, Kazantsev A, Apostol BL, Thompson LM, Yoder J, Aronin N and Di Figlia M: Autophagy regulates the processing of amino terminal huntingtin fragments. Hum Mol Genet 12: 3231-3244, 2003.

17. Fujita E, Kouroku Y, Isoai A, Kumagai H, Misutani A, Matsuda C, Hayashi YK and Momoi T: Two endoplasmic reticulum-associated degradation (ERAD) systems for the novel variant of the mutant dysferlin: ubiquitin/proteasome ERAD(I) and autophagy/lysosome ERAD(II). Hum Mol Genet 16: 618-629, 2007.

18. Park HK, Chu K, Jung KH, Lee ST, Bahn JJ, Kim M, Lee SK and Roh JK: Autophagy is involved in the ischemic preconditioning. Neurosci Lett 451: 16-19, 2009.

19. Sheng R, Zhang LS, Han R, Liu XQ, Gao B and Qin ZH: Autophagy activation is associated with neuroprotection in a rat model of focal cerebral ischemic preconditioning. Autophagy 6 : 482-494, 2010.

20. Lockshin RA and Zakeri Z: Apoptosis, autophagy, and more. Int J Biochem Cell Biol 36: 2405-2419, 2004.

21. Wen YD, Sheng R, Zhang LS, Han R, Zhang X, Zhang XD, Han F, Fukunaga K and Qin ZH: Neuronal injury in rat model of permanent focal cerebral ischemia is associated with activation of autophagic and lysosomal pathways. Autophagy 4: 762-769, 2008.

22. Koike M, Shibata M, Tadakoshi M, Gotoh K, Komatsu M, Waguri S, Kawahara N, Kuida K, Nagata S, et al: Inhibition of autophagy prevents hippocampal pyramidal neuron death after hypoxic-ischemic injury. Am J Pathol 172: 454-469, 2008.

23. Rami A, Langhagen A and Steiger S: Focal cerebral ischemia induces upregulation of Beclin 1 and autophagy-like cell death. Neurobiol Dis 29: 132-141, 2008.

24. Sadoshima J: The role of autophagy during ischemia/reperfusion. Autophagy 4: 402-403, 2008. 
25. Zheng YQ, Liu JX, Li XZ, Xu L and Xu YG: RNA interference-mediated downregulation of Beclin1 attenuates cerebral ischemic injury in rats. Acta Pharmacol Sin 30: 919-927, 2009.

26. Hou X, Zhang R, Lv H, Cai X, Xie G and Song X: Acupuncture at Baihui and Dazhui reduces brain cell apoptosis in heroin readdicts. Neural Regen Res 9: 164-70, 2014.

27. Hirai K, Aliev G,Nunomura A, Fujioka H, Russell RL, AtwoodCS Johnson AB, Kress Y, Vinters HV, et al: Mitochondrial abnormalities in Alzheimer's disease. J Neurosci 21: 3017-3023, 2001.

28. Qin ZH, Chen RW, Wang Y, Nakai M, Chuang DM and Chase TN: Nuclear factor $\kappa B$ nuclear translocation upregulates c-Myc and p53 expression during NMDA receptor-mediated apoptosis in rat striatum. J Neurosci 19: 4023-4033, 1999.

29. Longa EZ, Weinstein PR, Carlson S and Cummins R: Reversible middle cerebral artery occlusion without craniectomy in rats. Stroke 20: 84-91, 1989.

30. Rosen GD and Harry JD: Brain volume estimation from serial section measurements: a comparison of methodologies J Neurosci Methods 35: 115-124, 1990.

31. Napadow V, Makris N, Liu J, Kettner NW, Kwong KK and Hui KK: Effects of electroacupuncture versus manua acupuncture on the human brain as measured by fMRI. Hum Brain Mapp 24: 193-205, 2005.

32. Xiong LZ, Yang J, Wang Q and Lu ZH: Involvement of delta- and mu-opioid receptors in the delayed cerebral ischemic tolerance induced by repeated electroacupuncture preconditioning in rats. Chin Med J (Engl) 120: 394-399, 2007.

33. Talha S, Bouitbir J, Charles AL, Zoll J, Goette-Di Marco P, Meziani F, Piquard F and Geny B: Pretreatment with brain natriuretic peptide reduces skeletal muscle mitochondrial dysfunction and oxidative stress after ischemia-reperfusion. J Appl Physiol (1985) 114: 172-179, 2013.

34. Wu XD,DuLN,WuGCandCaoXD: Effects of electroacupuncture on blood-brain barrier after cerebral ischemia-reperfusion in rat. Acupunct Electrother Res 26: 1-9, 2001.

35. Zhao J, Xu H, Tian Y, Hu M and Xiao H: Effect of electroacupuncture on brain-derived neurotrophic factor mRNA expression in mouse hippocampus following cerebral ischemia-reperfusion injury. J Tradit Chin Med 33: 253-257, 2013.
36. Cui D, Wang L, Qi A, Zhou Q, Zhang X and Jiang W: Propofol prevents autophagic cell death following oxygen and glucose deprivation in PC12 cells and cerebral ischemia-reperfusion injury in rats. PLoS One 7: e35324, 2012.

37. Liu L, Fang YQ, Xue ZF, He YP, Fang RM and Li L: $\beta$-asarone attenuates ischemia-reperfusion-induced autophagy in rat brains via modulating JNK, p-JNK, Bcl-2 and Beclin 1. Eur J Pharmacol 680: 34-40, 2012.

38. Xia DY, Li W, Qian HR, Yao S, Liu JG and Qi XK: Ischemia preconditioning is neuroprotective in a rat cerebral ischemic injury model through autophagy activation and apoptosis inhibition. Braz J Med Biol Res 46: 580-588, 2013.

39. Wang JY, Xia Q, Chu KT, Pan J, Sun LN, Zeng B, Zhu YJ, Wang Q, Wang K and Luo BY: Severe global cerebral ischemia-induced programmed necrosis of hippocampal CA1 neurons in rat is prevented by 3-methyladenine: a widely used inhibitor of autophagy. J Neuropathol Exp Neurol 70: 314-22, 2011.

40. Liu C, Gao Y, Barrett J and Hu B: Autophagy and protein aggregation after brain ischemia. J Neurochem 115: 68-78, 2010.

41. Xu F, Li J, Ni W, Shen YW and Zhang XP: Peroxisome proliferator-activated receptor-gamma agonist 15d-prostaglandin J2 mediates neuronal autophagy after cerebral ischemia-reperfusion injury. PLoS One 8: e55080, 2013.

42. Xie R, Li X, Ling Y, Shen C, Wu X, Xu W and Gao X: $\alpha$-lipoic acid pre- and post-treatments provide protection against in vitro ischemia-reperfusion injury in cerebral endothelial cells via Akt/mTOR signaling. Brain Res 1482: 81-90, 2012.

43. Puyal J, Vaslin A, Mottier V and Clarke PG: Postischemic treatment of neonatal cerebral ischemia should target autophagy. Ann Neurol 66: 378-389, 2009

44. Zhang X, Yan H, Yuan Y, Gao J, Shen Z, Cheng Y, Shen Y, Wang RR, Wang X, et al: Cerebral ischemia-reperfusion-induced autophagy protects against neuronal injury by mitochondrial clearance. Autophagy 9: 1321-1333, 2013.

45. Zhu C, Wang X, Xu F, Bahr BA, Shibata M, Uchiyama Y, Hagberg $\mathrm{H}$ and Blomgren $\mathrm{K}$ : The influence of age on apoptotic and other mechanisms of cell death after cerebral hypoxia-ischemia. Cell Death Differ 12: 162-176, 2005. 